\title{
Finite Nucleus Model for the Fermi Contact Interaction
}

\author{
THORSTEINN HANNESSON AND S. M. BLINDER \\ Department of Chemistry, University of Michigan, Ann Arbor, Michigan 48109, U.S.A.
}

\begin{abstract}
s
A model for the Fermi contact interaction is proposed in which the charge and magnetic moment of the nucleus are uniformly distributed within a sphere of radius $r_{0}$. This leads to a Schrödinger equation, which is solvable without perturbation theory. In the mathematical limit $r_{0} \rightarrow 0$, the usual Coulomb plus delta function potential is obtained. It is shown that the magnetic perturbation energy goes to zero for a repulsive delta function, to $-\infty$ for an attractive delta function. A projected application is to computation of nuclear spin-spin coupling constants in molecules, particularly HD.

On propose un modèle pour l'interaction de contact de Fermi, dans lequel la charge et le moment magnétique du noyau sont distribués uniformément dans une sphère de rayon $r_{0}$. Ceci mène à une équation de Schrödinger qui est résoluble sans la théorie des perturbations. Dans la limite mathématique $r_{0} \rightarrow 0$ on obtient le potentiel de Coulomb ordinaire avec une fonction delta. Il est démontré que l'énergie de perturbation magnétique tend vers zéro pour une fonction delta répulsive et vers - $\infty$ pour une fonction delta attractive. Une application envisagée est un calcul des constantes de couplage spin-spin nucléaires dans les molécules, en particulier HD.

Ein Modell für die Fermi'sche Kontaktwechselwirkung wird vorgeschlagen, in welchem die Ladung und das magnetische Moment gleichförmig in einer Kugel mit Halbmesser $r_{0}$ verteilt werden. Dies führt zu einer Schrödingergleichung die ohne Störungstheorie lösbar ist. In der mathematischen Grenze $r_{0} \rightarrow 0$ wird das übliche Coulombpotential mit einer Deltafunktion erhalten. Es wird gezeigt, dass die magnetische Störungsenergie gegen Null für eine repulsive und gegen $-\infty$ für eine attraktive Deltafunktion konvergiert. Eine geplante Anwendung ist die Berechnung von nuklearen Spin-SpinKopplungskonstanten in Molekülen, besonders HD.
\end{abstract}

\section{Introduction}

The magnetic interaction between a nucleus and an atomic $s$ electron is most commonly represented by the Fermi contact interaction [1]

$$
\mathscr{H}^{\prime}=\frac{8}{3} \pi g g_{I} \mu_{B} \mu_{N}(\mathbf{S} \cdot \mathbf{I}) \delta^{3}(\mathbf{r})
$$

The simplest application of this formula is to the first-order magnetic energy in the $1 s$ state of atomic hydrogen $\left(Z=1, n=1, I=\frac{1}{2}\right)$ :

$$
\begin{gathered}
E_{F}^{(1)}=\left\langle\left|\mathscr{H}^{\prime}\right|\right\rangle=\lambda(\mathbf{S} \cdot \mathbf{I})_{F}=\lambda_{F} \\
\lambda \equiv \frac{8}{3} \pi g g_{I} \mu_{B} \mu_{N}\left|\psi_{1}(0)\right|^{2} \\
(\mathbf{S} \cdot \mathbf{I})_{F}=\frac{1}{2}\{F(F+1)-S(S+1)-I(I+1)\} \\
F=I \pm S=0 \text { or } 1
\end{gathered}
$$


The hyperfine splitting is then given by

$$
\nu_{\mathrm{HFS}}^{(1)}=\frac{E^{(1)}(F=1)-E^{(1)}(F=0)}{h}=\frac{\lambda}{h}
$$

Taking account of the reduced mass correction and the experimental freeelectron $g$ factor, the calculated first-order splitting has the numerical value

$$
\nu_{\mathrm{HFS}}^{(1)}=1420.4847 \mathrm{MHz}
$$

The corresponding experimental value is [2]

$$
\nu_{\mathrm{HFS}}=1420.4057517680(15) \mathrm{MHz}
$$

However, when Eq. (1) is used to calculate the second-order perturbation energy, the singularity in the operator causes $\nu_{\mathrm{HFS}}$ to diverge for $s$ states [3]. It is likewise necessary to consider second-order perturbation contributions from the Fermi contact interaction in the calculation of nuclear spin-spin coupling constants in molecules. This has led to divergences in certain such computations, notably for the HD molecule [4]. Such difficulties can be avoided by replacing the delta function in Eq. (1) by a function which does not have as strong a singularity [5]. In a model recently suggested by one of us [6], the nuclear magnetic moment is represented as a finite shell of magnetization, leading to a nonperturbative solution for the Schrödinger equation. In this paper we propose a physically more realistic model in which both the charge and magnetic moment of the nucleus are uniformly distributed within a sphere of radius $r_{0}$. We are not taking into account interactions other than those of the Coulomb and Fermi types. In particular, neither the $\mathbf{S} \cdot \mathbf{I}$ dipolar nor the $\mathbf{L} \cdot \mathbf{I}$ orbital nor the $A^{2}$ nuclear diamagnetic terms are to be considered. In fact, the latter corrections are known to make relatively minor contributions to the spin-spin coupling in HD [7].

\section{Finite Nucleus Potential}

For the nuclear charge distribution we set

$$
\rho(r)= \begin{cases}\rho_{0}, & r \leq r_{0} \\ 0, & r>r_{0}\end{cases}
$$

Normalizing to the total nuclear charge $+Z e$, using Gauss' theorem for the electrostatic potential for $r \leq r_{0}$, and assuming a Coulombic potential for $r>r_{0}$, we find the electrostatic potential

$$
V^{e}(r)= \begin{cases}-\left(Z e^{2} / 2 r_{0}^{3}\right)\left(3 r_{0}^{2}-r^{2}\right), & r \leq r_{0} \\ -Z e^{2} / r, & r>r_{0}\end{cases}
$$

The magnetic interaction is treated in an analogous fashion:

$$
V^{m}(r)= \begin{cases}\lambda_{F} \pi V_{0}, & r \leq r_{0} \\ 0, & r>r_{0}\end{cases}
$$


This approaches a delta function as $r_{0} \rightarrow 0$ if $V_{0}$ is chosen such that

$$
V^{m}(r)= \begin{cases}3 \lambda_{F} / 4 r_{0}^{3}, & r \leq r_{0} \\ 0, & r>r_{0}\end{cases}
$$

The potential due to the combined charge and magnetic moment of the nucleus is thus the sum of (7) and (9):

$$
V(r)=V^{e}(r)+V^{m}(r)
$$

We can now write the Schrödinger equation for a hydrogen atom with a finite proton. Using modified atomic units $(\hbar=e=\mu=1)$ and defining the radial function

$$
P(r)=r R(r)=(4 \pi)^{1 / 2} r \psi(r)
$$

we obtain

$$
\begin{array}{ll}
P^{\prime \prime}(r)+(2 / r) P(r)-k^{2} P(r)=0, & r>r_{0} \\
P^{\prime \prime}(r)+\left\{-k^{2}+3 / r_{0}-\frac{3}{2} \lambda_{F} / r_{0}^{3}-r^{2} / r_{0}^{3}\right\} P(r), & r \leq r_{0}
\end{array}
$$

where a wave number $k$ is defined by

$$
E=-k^{2} / 2
$$

Equations (12) and (13) for $s$ states $(l=0)$ are most easily solved in terms of confluent hypergeometric functions. For Eq. (12) we define

$$
z \equiv 2 k r, \quad \nu \equiv 1 / k, \quad P(r) \equiv W(z)
$$

Thus Eq. (12) is transformed into

$$
W^{\prime \prime}(z)+\left(\nu / z-\frac{1}{4}\right) W(z)=0
$$

which is Whittaker's equation in the notation of Buchholz [8]. The solution regular as $z \rightarrow \infty$ is

$$
P^{>}(r)=W_{\nu, 1 / 2}(z)
$$

Equation (13) is solved by the substitution

$$
x \equiv\left(4 / r_{0}^{3}\right)^{1 / 4} r ; \quad P^{<}(r) \equiv y(x)
$$

which transforms it into

$$
y^{\prime \prime}(x)-\left\{a+\frac{1}{4} x^{2}\right\} y(x)=0
$$

with

$$
a=-\left(-k^{2}+3 / r_{0}-\frac{3}{2} \lambda_{F} / r_{0}^{3}\right)\left(r_{0}^{3 / 2} / 2\right)
$$

This is Weber's differential equation. The solution regular as $x \rightarrow 0$ is [9]

$$
y(x)=y_{2}(a, x)=2 x e^{-x^{2} / 4}{ }_{1} F_{1}\left(\frac{1}{2} a+\frac{3}{4} ; \frac{3}{2} ; \frac{1}{2} x^{2}\right)
$$

This function is equivalent to $E_{\nu}^{(1)}(x)$ with $\nu=-\left(a+\frac{1}{2}\right)$ in the notation of Buchholz [10]. 
The eigenvalues given by $E=-1 / 2 \nu^{2}$ have the same form as those for the unperturbed Coulomb problem, except that the quantum numbers $\nu$ are no longer restricted to integral values. These eigenvalues are determined by a transcendental equation following from the requirement that the wave function and its derivative be continuous across $r=r_{0}$, that is,

$$
\left.\frac{P^{\prime>}(r)}{P^{>}(r)}\right|_{r_{0}^{+}}=\left.\frac{P^{<<}(r)}{P^{<}(r)}\right|_{r_{0}}
$$

\section{Results and Discussion}

For a hydrogen atom $\lambda$ is of the order $10^{-7}$, so the perturbation on the Coulombic potential is quite small. This means that the quantum number $\nu$ will differ only slightly from an integer. It is thus useful to define a quantum defect $\delta$ such that [6]

$$
\nu=n+\delta
$$

For the $1 s$ state of hydrogen, the energy can be expanded in terms of the quantum defect as follows:

$$
E_{F}=-1 / 2\left(1+\delta_{F}\right)^{2}=-\frac{1}{2}+\delta_{F}-\frac{3}{2} \delta_{F}^{2}+\cdots
$$

It is also advantageous to expand the Whittaker function to first order in the quantum defect:

$$
W_{\nu, 1 / 2}(2 r / \nu) \approx W_{\nu, 1 / 2}(2 r / n)+\left.\delta(\partial / \partial \nu)\left[W_{\nu, 1 / 2}(2 r / \nu)\right]\right|_{\nu=n}
$$

For $n=1$, this works out to

$$
W_{1+\delta_{F}, 1 / 2}(2 r / \nu) \approx 2 r e^{-r}\left[1+\delta_{F}\left(-1+r+\ln 2 r-\frac{1}{2} r\right)\right]
$$

Using the eigenvalue condition (22), we can now find $\delta_{F}$ as a function of $r_{0}$ in the physically significant range $r_{0} \sim 1 \mathrm{fm}=1 \times 10^{-13} \mathrm{~cm}=1.8897 \times 10^{-5} a_{0}$. For the exterior solution $P^{>}(r)\left(r>r_{0}\right)$

$$
\left.\frac{P^{\prime>}(r)}{P^{>}(r)}\right|_{r_{0}^{+}}=\frac{1-r_{0}+\delta_{F}\left(\frac{1}{2}+\left(1-r_{0}\right) \ln 2 r_{0}+3 r_{0}\right)}{r_{0}\left[1+\delta_{F}\left(-1+r_{0}+\ln 2 r_{0}-\frac{1}{2} r_{0}\right)\right]}
$$

For the interior solution $P^{<}(r)\left(r<r_{0}\right)$ it follows from Eqs. (18) and (21) that

$$
\begin{aligned}
\left.\frac{P^{<}(r)}{P^{<}(r)}\right|_{r_{\mathbf{0}}} & =\left.\left(\frac{4}{r_{0}^{3}}\right)^{1 / 4} \frac{d \ln y_{2}(a, x)}{d x}\right|_{x=x_{0}} \\
& =\left(\frac{4}{r_{0}^{3}}\right)^{1 / 4}\left\{\frac{1}{x_{0}}-\frac{x_{0}}{2}+\left.\frac{{ }_{1} F_{1}^{\prime}\left(\frac{1}{2} a+\frac{3}{4} ; \frac{3}{2} ; \frac{1}{2} x^{2}\right)}{{ }_{1} F_{1}\left(\frac{1}{2} a+\frac{3}{4} ; \frac{3}{2} ; \frac{1}{2} x^{2}\right)}\right|_{x=x_{0}}\right\}
\end{aligned}
$$

with $x_{0}=\left(4 r_{0}\right)^{1 / 4}$. Since $r_{0}$ is very small, we can approximate the hypergeometric function in Eq. (27) by its limiting form as $x \rightarrow 0$. This simplifies the equation to

$$
\left.\frac{P^{\prime<}(r)}{P^{<}(r)}\right|_{r_{0}} \approx\left(\frac{4}{r_{0}^{3}}\right)^{1 / 4}\left\{\frac{1}{x_{0}}+\frac{a}{3} x_{0}\right\} \approx \frac{1}{r_{0}}\left(1+\frac{\lambda_{F}}{2 r_{0}}\right)
$$


Solving for $\delta_{F}$ gives

$$
\delta_{F} \approx \frac{r_{0}+\lambda_{F} / 2 r_{0}}{\left(1+\lambda_{F} / 2 r_{0}\right)\left(1-\ln 2 r_{0}+\frac{1}{2} r_{0}\right)+\frac{1}{2}+\ln 2 r_{0}}
$$

These results can now be used to calculate the hyperfine splitting $\nu_{\mathrm{HFS}}$ in the $1 \mathrm{~s}$ state of hydrogen. Using Eq. (24) to first order in the quantum defect,

$$
\nu_{\mathrm{HFS}}=\frac{\delta_{1}-\delta_{0}}{h}
$$

Numerical values are given in Table $I$ for $r_{0}$ ranging from 0.1 to $10 \mathrm{fm}$. The experimental hyperfine splitting (5) would correspond to a proton radius $r_{0} \approx$ $7.5 \mathrm{fm}$, too large by at least a factor of 5 . Our model is not, however, intended to

TABLE I. Numerical values.

\begin{tabular}{llll}
\hline & $\delta_{1} \times 10^{8}$ & $\delta_{0} \times 10^{7}$ & $\nu$ hfs/MHz \\
\hline 0.1 & 5.30932 & 1.70793 & 1472.30 \\
0.2 & 5.35509 & 1.66277 & 1445.61 \\
0.3 & 5.37071 & 1.64816 & 1437.03 \\
0.4 & 5.37930 & 1.64088 & 1432.81 \\
0.5 & 5.38556 & 1.63646 & 1430.31 \\
0.6 & 5.38974 & 1.63347 & 1428.62 \\
0.8 & 5.39783 & 1.62959 & 1426.60 \\
1.0 & 5.40555 & 1.62690 & 1425.34 \\
1.5 & 5.42668 & 1.62233 & 1423.72 \\
2.0 & 5.45411 & 1.61812 & 1422.76 \\
5.0 & 5.75969 & 1.58533 & 1421.29 \\
7.0 & 6.09869 & 1.55022 & 1420.49 \\
10.0 & 6.84251 & 1.47661 & 1421.00 \\
\hline
\end{tabular}

provide a highly accurate representation of the hydrogen atom itself. More detailed consideration of nuclear size effects within the context of the relativistic theory is given in the papers of Zemach [11], Idding and Platzman [12], Hockensmith and Foldy [13], and Moore and Moss [14].

It is amusing, nonetheless, to apply our numerical results to a quark model of the proton. A proton is made of two $u$ quarks (charge $+\frac{2}{3}$ ) and one $d$ quark (charge $-\frac{1}{3}$ ). Assuming a total $u$ magnetic moment of $\left(\frac{4}{3}\right) \mu_{P}$ and a $d$ magnetic moment of 
$-\left(\frac{1}{3}\right) \mu_{P}$, the experimental value of $\nu_{\mathrm{HFS}}$ could be accounted for by the radii $r_{u} \approx 1.4 \mathrm{fm}, r_{d} \approx 0.35 \mathrm{fm}$. These latter parameters are reasonably in accord with the charge distribution in a proton as determined from high-energy electronproton scattering [15].

\section{Limiting Behavior as $\boldsymbol{r}_{\mathbf{0}} \rightarrow 0$}

In the limit as the nucleus is reduced to a point charge with point magnetic dipole, the operator (9) becomes a delta function and the conventional Fermi contact interaction is regained. For the $F=1$ state $\left(\lambda_{F}>0\right)$, Eq. (30) converges uniformly to zero as $r_{0} \rightarrow 0$. Thus the energy approaches its unperturbed Coulomb value. Figure 1 shows the quantum defect as a function of $r_{0}$.

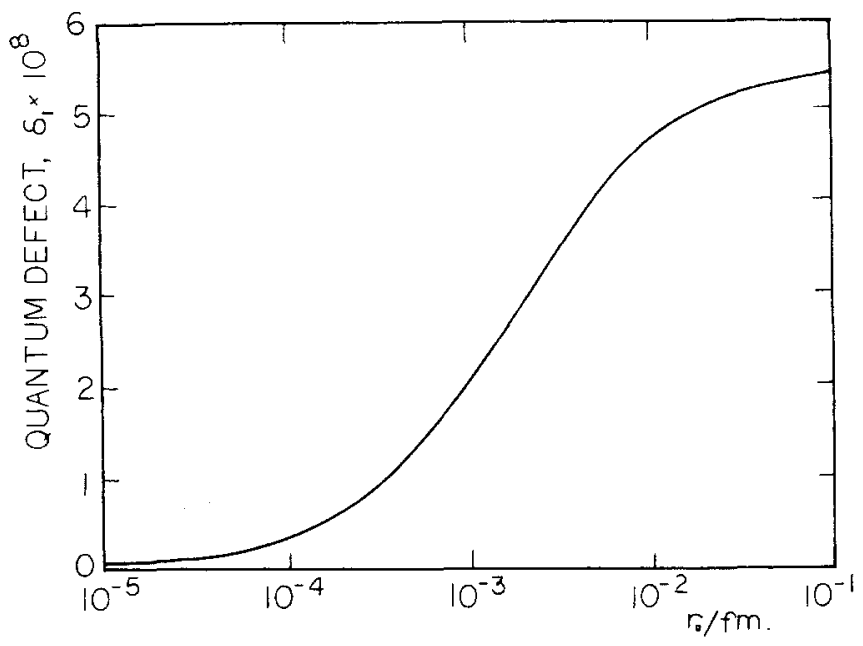

Figure 1. Quantum defect for the $F=1$ state of hydrogen.

For the $F=0$ state $\left(\lambda_{F}<0\right)$, Eq. (30) implies $\delta_{F} \approx O(1)$ in the vicinity of $r_{0} \approx\left|\lambda_{F}\right| / 2$ (Fig. 2). Thus Eqs. (26) and (29) become invalid, being based on the assumption that $\delta \ll 1$. The $r_{0} \rightarrow 0$ limit for $\lambda_{F}<0$ can be found instead by the following argument. For $r_{0}$ sufficiently small, the electrostatic terms in (13) can be neglected and

$$
P^{\prime \prime}(r)+\left[\frac{3}{2}\left|\lambda_{F}\right| / r_{0}^{3}-k^{2}\right] P(r) \approx 0
$$

with the changes of variables

$$
z \equiv 2 k r, \quad z_{0} \equiv 2 k r_{0}, \quad P(r) \equiv Z(z)
$$

we obtain

$$
Z^{\prime \prime}(z)+\left[\frac{3\left|\lambda_{F}\right| k}{z_{0}^{3}}-\frac{1}{4}\right] Z(z) \approx 0
$$




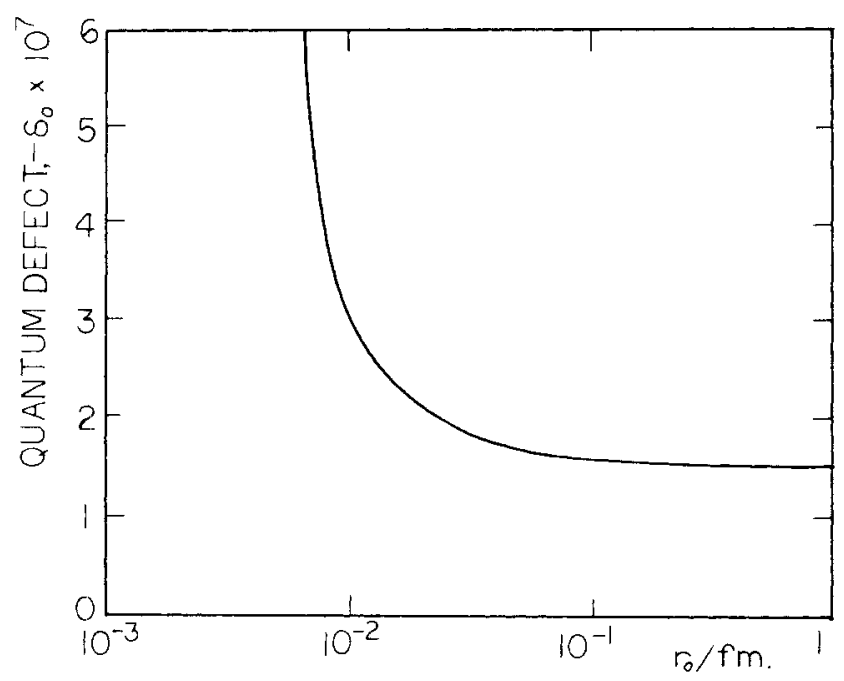

Figure 2. Quantum defect for the $F=0$ state of hydrogen.

The solution regular at $z=0$ is

$$
Z(z)=\sin \left[\left(\frac{3\left|\lambda_{F}\right| k}{z_{0}^{3}}-\frac{1}{4}\right)^{1 / 2} z\right]
$$

Thus

$$
\left.\frac{Z^{\prime}(z)}{Z(z)}\right|_{z=z_{0}} \approx\left(\frac{3\left|\lambda_{F}\right| k}{z_{0}^{3}}-\frac{1}{4}\right)^{1 / 2} \operatorname{cotan}\left[\left(\frac{3\left|\lambda_{F}\right| k}{z_{0}^{3}}-\frac{1}{4}\right)^{1 / 2} z_{0}\right]
$$

Consider now asymptotic forms as $z_{0} \rightarrow \infty$. For the exterior solution [16]

$$
W_{\nu, 1 / 2}\left(z_{0}\right) \sim z_{0}^{\nu} e^{-z_{0} / 2}
$$

and

$$
\left.\frac{W_{\nu, 1 / 2}^{\prime}(z)}{W_{\nu, 1 / 2}(z)}\right|_{z=z_{0}} \approx \frac{\nu}{z_{0}}-\frac{1}{2} \approx-\frac{1}{2}
$$

The continuity condition (22) is thus approximated by

$$
\left[\left(\frac{3\left|\lambda_{F}\right| k}{z_{0}^{3}}-\frac{1}{4}\right)^{1 / 2} z_{0}\right] \operatorname{cotan}\left[\left(\frac{3\left|\lambda_{F}\right| k}{z_{0}^{3}}-\frac{1}{4}\right)^{1 / 2} z_{0}\right] \approx-\frac{1}{2} z_{0}
$$

The limit $z_{0} \rightarrow \infty$ is approached as the argument of the cotangent approaches $\pi$ $(\operatorname{cotan} \pi=-\infty)$. We find thus

$$
\left(\frac{3\left|\lambda_{F}\right| k}{z_{0}^{3}}-\frac{1}{4}\right)^{1 / 2} z_{0} \approx \pi
$$


and

$$
k^{2} \approx \frac{3\left|\lambda_{F}\right|}{2 r_{0}^{3}}-\frac{\pi^{2}}{r_{0}^{2}} \approx \frac{3\left|\lambda_{F}\right|}{2 r_{0}^{3}}
$$

Since $z_{0}=2 k r_{0} \approx\left(6\left|\lambda_{F}\right| / r_{0}\right)^{1 / 2}$, it is verified that the limit $z_{0} \rightarrow \infty$ does indeed correspond to $r_{0} \rightarrow 0$. Therefore, $E=-k^{2} / 2$ approaches $-\infty$ as $r_{0} \rightarrow 0$ in the $F=0$ state. This limiting behavior is, in fact, identical with that for an attractive three-dimensional square-well potential. Our conclusions on the energies of a hydrogen atom perturbed by attractive and repulsive delta function potentials are in accord with those of Velenic et al. [17], based on variational calculations.

\section{Bibliography}

[1] E. Fermi, Z. Phys. 60, 320 (1930); S. M. Blinder, Adv. Quantum Chem. 2 , 47 (1965).

[2] N. F. Ramsey, Natl. Bur. Stand. (U.S.A.) Special Publication 465 (1977), p. 14.

[3] C. Schwartz, Ann. Phys. (N.Y.) 2156 (1959).

[4] T. P. Das and R. Bersohn, Phys. Rev. 115, 897 (1959); H. Wrubel and J. Voitländer, Mol. Phys. 25, 323 (1973).

[5] J. D. Power and R. M. Pitzer, Chem. Phys. Lett. 8, 615 (1971); W. Sanger and J. Voitländer, Z. Naturforsch. 29a, 1866 (1973); M. J. Gregson, G. G. Hall, and D. Rees, J. Phys. B 3, 1195 (1970).

[6] S. M. Blinder, Phys. Rev. A 18, 853 (1978).

[7] E. Ishiguro, Phys. Rev. 111, 203 (1958).

[8] H. Buchholz, The Confluent Hypergeometric Function (Springer, New York, 1969).

[9] Handbook of Mathematical Functions (Natl. Bur. Stand. U.S.A., Washington, D.C., 1972), p. 686.

[10] H. Buchholz, in Ref. 8, p. 40.

[11] A. C. Zemach, Phys. Rev. 104, 1771 (1956).

[12] C. K. Idding and P. M. Platzman, Phys. Rev. 113, 102 (1959); 115, 919 (1959).

[13] D. A. Hockensmith and L. L. Foldy, Phys. Rev. 133, A1514 (1964).

[14] E. A. Moore and R. E. Moss, Mol. Phys. 30, 1297 (1975); 30, 1315 (1975).

[15] D. N. Olson, H. F. Schopper, and R. R. Wilson, Phys. Rev. Lett. 6, 286 (1961).

[16] H. Buchholz, in Ref. 8, p. 90.

[17] A. Velenik, T. Zivković, W. H. de Jeu, and J. N. Murrell, Mol. Phys. 18, 693 (1970).

Received May 16, 1978

Revised July 18, 1978

Accepted for publication August 29, 1978 